\title{
Predictive role of human equilibrative nucleoside transporter 1 in patients with pancreatic cancer treated by curative resection and gemcitabine-only adjuvant chemotherapy
}

\author{
TORU AOYAMA ${ }^{1 *}$, KEISUKE KAZAMA $^{1 *}$, YOHEI MIYAGI ${ }^{2}$, MASAAKI MURAKAWA ${ }^{1}$, \\ KOICHIRO YAMAOKU ${ }^{1}$, YOSUKE ATSUMI ${ }^{1}$, MANABU SHIOZAWA ${ }^{1}$, MAKOTO UENO ${ }^{3}$, \\ MANABU MORIMOTO $^{3}$, TAKASHI OSHIMA ${ }^{4}$, NORIO YUKAWA ${ }^{4}$, TAKAKI YOSHIKAWA ${ }^{4}$, \\ YASUSHI RINO ${ }^{4}$, MUNETAKA MASUDA $^{4}$ and SOICHIRO MORINAGA ${ }^{1}$ \\ ${ }^{1}$ Department of Gastrointestinal Surgery; ${ }^{2}$ Molecular Pathology and Genetics Division; \\ ${ }^{3}$ Department of Hepatobiliary Pancreatic Oncology, Kanagawa Cancer Center, Yokohama, \\ Kanagawa 241-8515; ${ }^{4}$ Department of Surgery, Yokohama City University, Yokohama, Kanagawa 236-0027, Japan
}

Received October 21, 2015; Accepted February 23, 2017

DOI: $10.3892 / \mathrm{ol} .2017 .6220$

\begin{abstract}
The predictive roles of human equilibrative nucleoside transporter 1 (hENT-1) in patients who undergo curative resection and adjuvant chemotherapy with gemcitabine alone have not been established. The present study retrospectively analyzed the clinical data from 101 consecutive patients who underwent curative resection and who received gemcitabine adjuvant chemotherapy for the treatment of pancreatic cancer at Kanagawa Cancer Center (Yokohama, Japan) between 2005 and 2014. The associations between the hENT-1 status and the survival and clinicopathological features of the patients were investigated. Of the 101 patients, 60 patients (59.4\%) had high levels of hENT-1 expression. A significant association was observed between hENT-1 status and sex; however, for all the other clinicopathological parameters, including tumor and node stages, no differences were observed between the high and low hENT-1 expression groups. The median follow-up period of the present study was 67.3 months. Between the high and low hENT-1 expression groups, there was a statistically significant difference in the 5-year overall survival (OS) rates following surgery (20.6 and 8.9\%, respectively; $\mathrm{P}=0.019)$. In addition, a significant difference was observed in the recurrence-free survival (RFS) rates at 5 years following surgery $(\mathrm{P}=0.049)$. hENT-1 status was one of the important predictive factors for OS and RFS in patients with pancreatic
\end{abstract}

Correspondence to: Dr Toru Aoyama, Department of Gastrointestinal Surgery, Kanagawa Cancer Center, 2-3-2 Nakao, Yokohama, Kanagawa 241-8515, Japan

E-mail: aoyamat@kcch.jp

*Contributed equally

Key words: pancreatic cancer, human equilibrative nucleoside transporter 1 , adjuvant chemotherapy, gemcitabine cancer who underwent curative resection followed by adjuvant chemotherapy with gemcitabine. Adjuvant chemotherapy with gemcitabine alone may be insufficient, particularly in patients with certain relevant risk factors.

\section{Introduction}

Pancreatic cancer, which has a 5-year survival rate of $<5 \%$, is a major cause of cancer- associated mortality worldwide $(1,2)$. Complete resection is essential for the cure of pancreatic cancer. However, only $10-20 \%$ of patients with pancreatic cancer are candidates for curative resection $(3,4)$. Furthermore, due to the high rate of recurrence, the postoperative 5-year survival rate is only $10-20 \%$ when curative resection is performed (3-5).

Numerous randomized controlled studies of adjuvant chemotherapy following pancreatic cancer resection have been conducted (6-8). Recently, the European Study Group for Pancreatic Cancer-1 and -3 trials and the Charite Onkologic 001 (CONKO-001) trial demonstrated that treatment with gemcitabine or fluorouracil (FU) plus folinic acid could significantly increase overall survival (OS) time following surgical resection in patients with pancreatic cancer, as compared with surgery alone (6-8). Based on these results, adjuvant chemotherapy with gemcitabine is now considered to be a standard treatment and is routinely recommended following curative resection for pancreatic cancer. However, even adjuvant chemotherapy with gemcitabine is unable to completely prevent the development of recurrence. The selection of patients who can derive a true benefit from gemcitabine may be an important step towards improving the clinical outcomes associated with pancreatic cancer.

Human equilibrative nucleoside transporter-1 (hENT-1) is a nucleoside transporter protein that mediates the entry of cytotoxic chemotherapies into cells, including gemcitabine (9). In vitro studies have demonstrated that the expression of hENT-1 is associated with sensitivity to nucleoside analogues (10). Specifically, the overexpression of hENT-1 can enhance response to gemcitabine in human pancreatic cancer, 
whereas cells in which hENT-1 expression is absent are resistant to gemcitabine $(11,12)$. Previous studies of patients with pancreatic cancer treated with gemcitabine indicate that there is an association between hENT-1 expression and treatment outcome $(13,14)$. In addition, clinical studies of adjuvant chemoradiation therapy for resected pancreatic cancer have revealed that, compared with low hENT-1 expression, a high tumor expression level of hENT-1 is associated with a longer patient survival time following gemcitabine chemotherapy $(15,16)$. However, few published studies have evaluated the predictive value of hENT-1 expression in patients with pancreatic cancer treated by resection and gemcitabine-only adjuvant chemotherapy, and no definite conclusions can be made regarding the predictive value of hENT-1 in such patients.

The characterization of genes associated with sensitivity or resistance to antitumor agents, using cancer tissues from individual patients, serves a critical role in the development and provision of individualized adjuvant chemotherapy treatments. In the present study, hENT-1 expression was investigated in consecutive patients who underwent curative resection followed by adjuvant chemotherapy with gemcitabine, and the associations between hENT-1 expression, clinicopathological parameters and survival were investigated.

\section{Materials and methods}

Patient selection. The patients were selected from the medical records of 201 consecutive patients who underwent pancreatic surgery at Kanagawa Cancer Center (Yokohama, Japan) between April 2005 and December 2014. The following inclusion criteria were applied: The patients had a pathologically common type of pancreatic adenocarcinoma according to the definitions of the International Union Against Cancer (UICC) TNM 7th edition (17); the patients initially underwent curative resection and the resected specimens were archived; and the patients received adjuvant chemotherapy with gemcitabine. The resected specimens were examined histopathologically and were staged according to the UICC TNM 7th edition (17). Patients with other pancreatic and periampullary neoplasms, including intraductal papillary mucinous neoplasm, cystadenocarcinoma and endocrine tumors, as well as patients who underwent R2 resection, were excluded from the present study. The present study was approved by the Institutional Review Board of the Kanagawa Cancer Center.

In total, 101 patients were eligible for inclusion in the present study, including 57 men and 44 women, with a median age of 66 years.

Surgical procedure. All pancreatic surgeries were performed in accordance with standardized procedures previously described (18-20). Briefly, in cases of distal pancreatectomy, lymph node dissection was performed in the region of the celiac trunk and the superior mesenteric artery and vein, as well as behind the pancreas along the left side of the renal vein and the left adrenal gland. In each case, intraperitoneal drains were placed close to the pancreatic anastomosis and stump. In cases of pancreaticoduodenectomy, subtotal stomach-preserving pancreaticoduodenectomy was performed as the standard procedure. Lymph node dissection along the hepatoduodenal ligament, common hepatic artery, vena cava, superior mesenteric vein and the right side of the superior mesenteric artery were included in the procedure as standard. Multiple intraperitoneal drains were placed, with the first being posterior to the hepaticojejunostomy and the second on the anterior surface of the pancreaticojejunostomy or the closed remnant of the pancreas.

Adjuvant chemotherapy. Gemcitabine treatment was initiated within 8 weeks following surgery. The patients received a weekly dose of $1,000 \mathrm{mg} / \mathrm{m}^{2}$ for 3 weeks, followed by 1 week of rest. The gemcitabine treatment was continued for 6 months.

Follow-up. Patients were followed up at outpatient clinics. Hematological tests and physical examinations were performed a minimum of every 2 weeks during adjuvant chemotherapy, and at least every 3 months for 5 years after course of adjuvant chemotherapy had been completed. The levels of the tumor markers carcinoembryonic antigen and carbohydrate antigen 19-9 were assessed at least every 3 months for 5 years.

Patients underwent a computed tomography examination every 3 months during the first 3 years following surgery, and then every 6 months until 5 years following surgery. Peritoneal recurrence was defined as positive when at least one of the following findings was identified on imaging studies: Massive ascites, ascites confirmed by cytology, enhanced abdominal nodules, abnormal intestinal wall thickness, increased fat density of the intestinal mesentery, diffuse hydronephrosis or an intraabdominal mass. Imaging studies were checked by one radiologist and two staff physicians. When liver metastasis was suspected based on imaging studies, gadolinium-ethoxybenzyl-diethylenetriamine-pentaacetate-enhanced magnetic resonance imaging or contrast-enhanced ultrasonography was performed to confirm the diagnosis.

Immunohistochemical analysis of hENT-1 expression. Hematoxylin and eosin-stained slides containing specimens from each pancreatic adenocarcinoma were reviewed, and a representative tumor region and the corresponding formalin-fixed, paraffin-embedded tissue block were selected for use in a tissue microarray. The hENT-1 expression was evaluated using a rabbit anti-hENT-1 monoclonal antibody (MBL International Co., Woburn, MA, USA). The immunohistochemical staining procedure is described elsewhere (21).

The staining intensity for the hENT-1 protein and the percentage of positive tumor cells were scored, and a composite score (hENT-1 score) was obtained by calculating the sum of these two scores. The staining intensity for the hENT-1 protein was assigned a score ranging from 0 to 3 : $0+$, no staining; 1+, weakly positive; $2+$, moderately positive; and $3+$, strongly positive. The percentage of positive tumor cells was scored as follows: $0+$, no positive tumor cells; $1+,<50 \%$ positive cells; $2+, 50-80 \%$ positive cells; and $3+, \geq 81 \%$ positive cells. Tumors with composite hENT-1 scores of 0-3 were classified as having low hENT-1 expression and tumors with scores of 4-6 as having high hENT-1 expression.

Evaluations and statistical analysis. The significance of the associations between hENT-1 expression and clinicopathological parameters were determined using Fisher's exact test or $\chi^{2}$ test. OS was defined as the period between surgery 
Table I. Associations between patient characteristics and hENT-1 expression.

\begin{tabular}{|c|c|c|c|}
\hline Factor & High hENT-1 group, n (\%) & Low hENT-1 group, n (\%) & P-value \\
\hline Total & 60 & 41 & \\
\hline Sex & & & 0.017 \\
\hline Male & $28(46.7)$ & $29(70.7)$ & \\
\hline Female & $32(53.3)$ & $12(29.3)$ & \\
\hline Age, years & & & 0.725 \\
\hline$<65$ & $27(45.0)$ & $17(41.5)$ & \\
\hline$\geq 65$ & $33(55.0)$ & $24(58.5)$ & \\
\hline Tumor location & & & 0.536 \\
\hline Pancreatic head & $42(70.0)$ & $31(75.6)$ & \\
\hline Pancreatic body/tail & $18(30.0)$ & $10(24.4)$ & \\
\hline Pathological differentiation & & & 0.370 \\
\hline Well differentiated & $51(85.0)$ & $32(78.0)$ & \\
\hline Moderately/poorly differentiated & $9(15.0)$ & $9(22.0)$ & \\
\hline UICC pT factor & & & 0.142 \\
\hline $\mathrm{T} 1 / \mathrm{T} 2$ & $6(10.0)$ & $1(2.4)$ & \\
\hline $\mathrm{T} 3$ & $54(90.0)$ & 40 (97.6) & \\
\hline Lymph node metastasis & & & 0.871 \\
\hline N0 & $14(23.3)$ & $9(22.0)$ & \\
\hline N1 & $46(76.7)$ & $32(78.0)$ & \\
\hline Lymphatic invasion & & & 0.496 \\
\hline No & $29(48.3)$ & $17(41.5)$ & \\
\hline Yes & $31(51.7)$ & $24(58.5)$ & \\
\hline Vascular invasion & & & 0.590 \\
\hline No & $16(26.7)$ & $9(22.0)$ & \\
\hline Yes & $44(73.3)$ & $32(78.0)$ & \\
\hline
\end{tabular}

hENT-1, human equilibrative nucleoside transporter 1; UICC, International Union Against Cancer.

and mortality. Recurrence-free survival (RFS) was defined as the period between surgery and recurrence or mortality, whichever came first. The data of the patients who had not experienced an event were censored at the date of the final observation. The OS and RFS were evaluated by univariate and multivariate analyses. OS and RFS curves were calculated using the Kaplan-Meier method and compared using the log-rank test. The univariate and multivariate survival analyses were performed using Cox's proportional hazards model. $\mathrm{P}<0.05$ was considered to indicate a statistically significant difference. The survival data were obtained from hospital records or from the city registry system. SPSS software (v11.0J Win; SPSS, Inc., Chicago, IL, USA) was used for all of the statistical analyses.

\section{Results}

Patients. A total of 201 patients underwent surgical resection between 2005 and 2014. Of these patients, 101 were eligible for inclusion in the present study. The ages of the patients ranged from 40-78 years (median, 66 years); 57 patients were male and 44 were female. A total of 28 patients underwent distal pancreatectomy, 70 underwent pancreaticoduodenectomy and
3 underwent total pancreatic resection. In total, 85 patients achieved R0 resection and 16 patients achieved R1 resection. The median follow-up period was 67.3 months (range, 22.2-122.7 months).

Immunohistochemical analyses. Representative results from the immunohistochemical staining for hENT-1 in pancreatic adenocarcinoma tissue sections are shown in Fig. 1. Immunoreactivity was observed in the cytoplasm of cancer cells. Among the 101 tumor samples, 38 samples exhibited negative staining, 11 samples exhibited weak staining, 32 samples exhibited moderate staining and 20 samples exhibited strong staining. Of the 63 tumor samples that showed positive staining, 3 samples had $<50 \%$ positive cells, 10 samples had $50-80 \%$ positive cells and 50 samples had $\geq 81 \%$ positive cells. According to the hENT-1 scoring system, 41 patients were assigned to the low hENT-1 expression group and 60 patients to the high hENT-1 expression group.

Association between clinicopathological factors and hENT-1 expression. A total of eight clinicopathological factors were compared between the patients with high and low hENT-1 


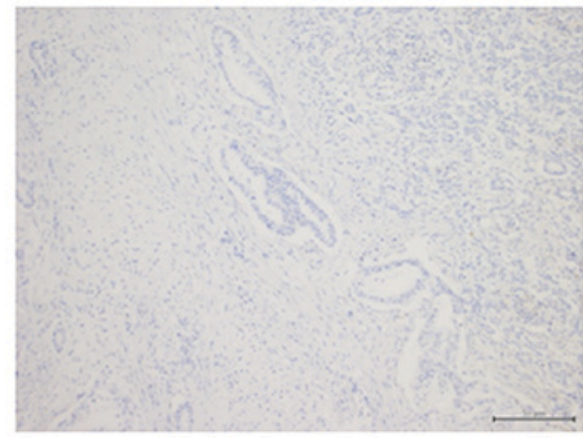

Immunohistochemistry score 0

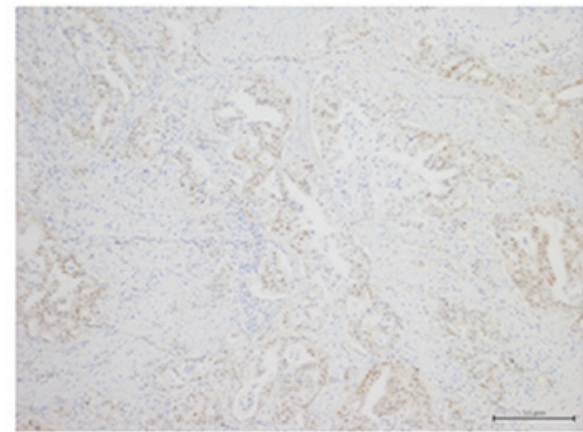

Immunohistochemistry score 1+

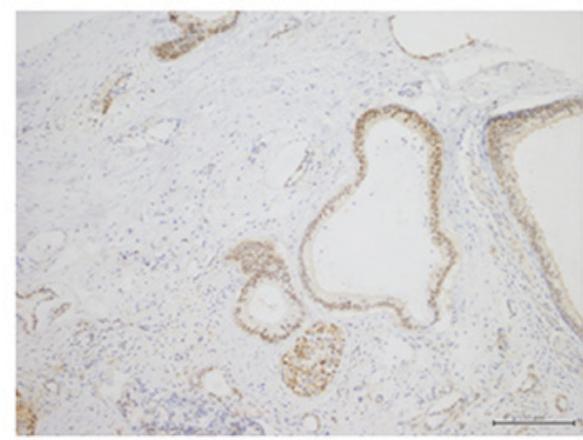

Immunohistochemistry score 2+

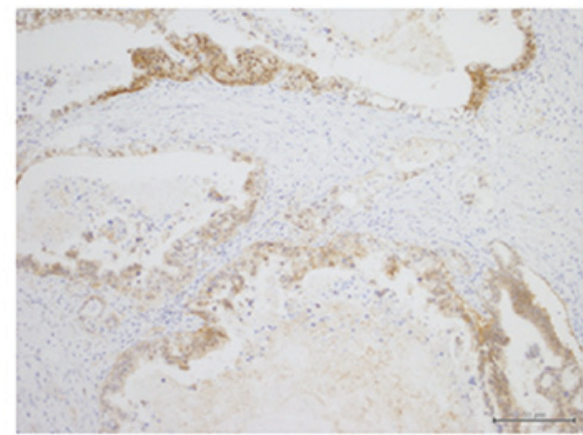

Immunohistochemistry score $3+$

Figure 1. Representative results of the immunohistochemical staining of human equilibrative nucleoside transporter 1 . Scale bar=100 $\mu \mathrm{m}$.

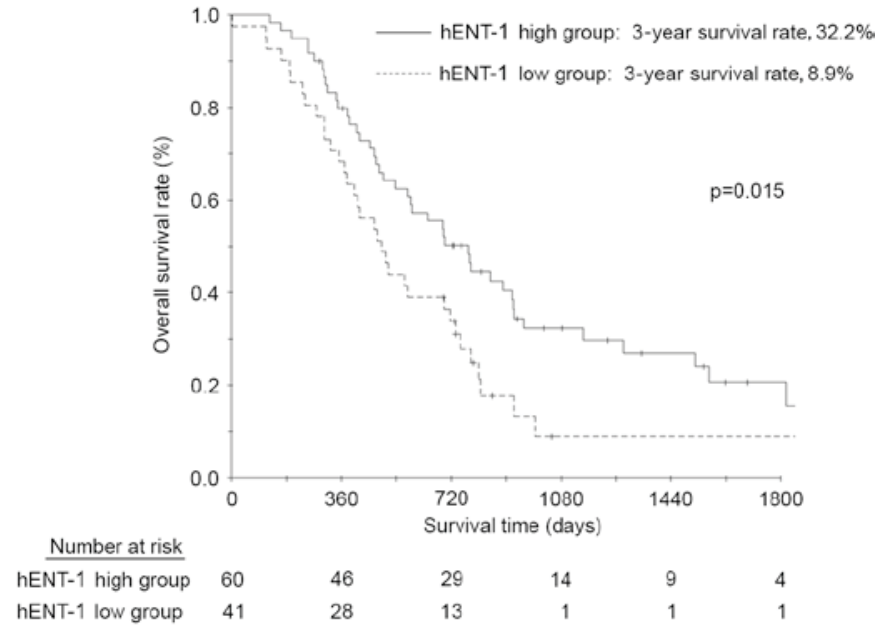

Figure 2. Kaplan-Meier curves and log-rank test for overall survival rates in the hENT-1 high and hENT-1 low groups. hENT-1, human equilibrative nucleoside transporter 1 .

expression. Although a significant difference was observed in sex distribution, no differences were observed between the two groups for all other clinicopathological parameters (Table I).

Survival analysis. The OS rates at 3 and 5 years following surgery in the patients with high hENT-1 expression were 32.2 and $20.6 \%$, respectively; and 8.9 and $8.9 \%$ in the patients with low hENT-1 expression (Fig. 2); this result was also statistically significant $(\mathrm{P}=0.015)$. The multivariate analyses demonstrated

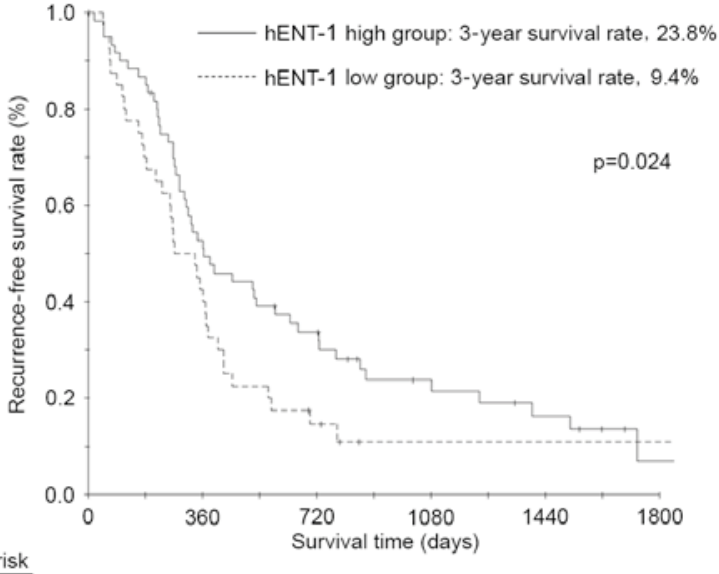

Number at risk

hENT-1 high group 60 hENT-1 low group 41

Figure 3. Kaplan-Meier curves and log-rank test for recurrence-free survival rates in the hENT-1 high and hENT-1 low groups. hENT-1, human equilibrative nucleoside transporter 1 .

that hENT-1 expression status and lymphatic invasion were significant risk factors for OS (Table II).

The RFS rates at 3 and 5 years following surgery in the patients with high hENT-1 expression were 23.8 and $6.8 \%$, respectively; and 9.4 and $9.4 \%$ in the patients with low hENT-1 expression (Fig. 3). The difference in both 3 and 5-year survival rates was statistically significantly $(\mathrm{P}=0.024)$. The multivariate analyses demonstrated that hENT-1 expression status, resection status and lymphatic invasion were significant risk factors for RFS (Table III). 
Table II. Univariate and multivariate analyses of risk factors for overall survival.

\begin{tabular}{|c|c|c|c|c|c|c|c|}
\hline \multirow[b]{2}{*}{ Factor } & \multirow[b]{2}{*}{$\mathrm{n}$} & \multicolumn{3}{|c|}{ Univariate analysis } & \multicolumn{3}{|c|}{ Multivariate analysis } \\
\hline & & OR & $95 \%$ CI & P-value & OR & $95 \% \mathrm{CI}$ & P-value \\
\hline Sex & & & & 0.561 & & & - \\
\hline Female & 44 & 1.000 & - & & - & - & \\
\hline Male & 57 & 1.143 & $0.728-1.795$ & & - & - & \\
\hline Age, years & & & & 0.740 & & & - \\
\hline$<65$ & 44 & 1.000 & - & & - & - & \\
\hline$\geq 65$ & 57 & 1.081 & $0.683-1.709$ & & - & - & \\
\hline Resection status & & & & 0.041 & & & - \\
\hline R0 & 85 & 1.000 & - & & - & - & \\
\hline R1 & 16 & 1.850 & $1.026-3.336$ & & - & - & \\
\hline Tumor location & & & & 0.024 & & & 0.057 \\
\hline Pancreatic body/tail & 28 & 1.000 & - & & 1.000 & - & \\
\hline Pancreatic head & 73 & 1.840 & $1.085-3.120$ & & 1.667 & $0.984-2.825$ & \\
\hline Pathological differentiation & & & & 0.892 & & & - \\
\hline Well differentiated & 83 & 1.000 & - & & - & - & \\
\hline $\begin{array}{l}\text { Moderately/poorly } \\
\text { differentiated }\end{array}$ & 18 & 1.042 & $0.572-1.898$ & & - & - & \\
\hline UICC pT factor & & & & 0.035 & & & - \\
\hline $\mathrm{T} 1 / \mathrm{T} 2$ & 7 & 1.000 & - & & - & - & \\
\hline $\mathrm{T} 3$ & 94 & 4.545 & $1.113-18.559$ & & - & - & \\
\hline Lymph node metastasis & & & & 0.038 & & & - \\
\hline N0 & 23 & 1.000 & - & & - & - & \\
\hline N1 & 78 & 1.802 & $1.034-3.140$ & & - & - & \\
\hline Lymphatic invasion & & & & 0.001 & & & 0.001 \\
\hline No & 46 & 1.000 & - & & 1.000 & - & \\
\hline Yes & 55 & 2.192 & $1.374-3.498$ & & 2.250 & $1.405-3.603$ & \\
\hline Vascular invasion & & & & 0.032 & & & - \\
\hline No & 38 & 1.000 & - & & - & - & \\
\hline Yes & 63 & 1.678 & $1.044-2.695$ & & - & - & \\
\hline hENT-1 status & & & & 0.021 & & & 0.019 \\
\hline High & 60 & 1.000 & - & & 1.000 & - & \\
\hline Low & 41 & 1.713 & $1.083-2.707$ & & 1.740 & $1.095-2.766$ & \\
\hline
\end{tabular}

hENT-1, human equilibrative nucleoside transporter 1; OR, odds ratio; CI, confidence interval; UICC, International Union Against Cancer.

\section{Discussion}

The present study evaluated hENT-1 expression status in patients with pancreatic adenocarcinoma who underwent curative resection followed by adjuvant chemotherapy with gemcitabine, and identified $60 \%$ of these patients as exhibiting high hENT-1 expression. The OS and RFS rates of the patients differed significantly based on their hENT-1 status. The present results indicated that gemcitabine alone was insufficient as an adjuvant therapy, particularly in the patients with low hENT-1 expression. These patients may be a target group for clinical trials of novel treatments.

A predictive role of hENT-1 in pancreatic cancer has been reported in previous studies. Giovannetti et al (13) characterized the expression patterns of genes involved in gemcitabine activity in pancreatic tumor specimens from surgical or biopsy samples of 102 patients with pancreatic cancer treated with gemcitabine, and evaluated their associations with treatment outcomes. The authors reported that the patients with increased levels of hENT-1 expression had significantly longer OS and disease-free survival (DFS) times and times-to-progression compared with those with lower transcriptional hENT-1 levels. In addition, Spratlin et al (14) reported that patients who had uniformly detectable hENT-1 immunostaining in their pancreatic adenocarcinoma samples had a significantly longer survival time following gemcitabine chemotherapy compared with hENT-1 status. Similar results were observed in the adjuvant setting (21). 
Table III. Univariate and multivariate analyses of risk factors for recurrence-free survival.

\begin{tabular}{|c|c|c|c|c|c|c|c|}
\hline \multirow[b]{2}{*}{ Factor } & \multirow[b]{2}{*}{$\mathrm{n}$} & \multicolumn{3}{|c|}{ Univariate analysis } & \multicolumn{3}{|c|}{ Multivariate analysis } \\
\hline & & OR & $95 \%$ CI & P-value & OR & $95 \%$ CI & P-value \\
\hline Sex & & & & 0.874 & & & - \\
\hline Female & 44 & 1.000 & - & & - & - & \\
\hline Male & 57 & 1.035 & $0.674-1.590$ & & - & - & \\
\hline Age, years & & & & 0.293 & & & - \\
\hline$<65$ & 44 & 1.000 & - & & - & - & \\
\hline$\geq 65$ & 57 & 1.264 & $0.817-1.954$ & & - & & - \\
\hline Resection status & & & & 0.001 & & & 0.036 \\
\hline $\mathrm{R} 0$ & 85 & 1.000 & - & & 1.000 & - & \\
\hline R1 & 16 & 2.668 & $1.469-4.845$ & & 1.931 & $1.044-3.572$ & \\
\hline Tumor location & & & & 0.016 & & & - \\
\hline Pancreatic body/tail & 28 & 1.000 & - & & - & - & \\
\hline Pancreatic head & 73 & 1.816 & $1.118-2.949$ & & - & - & \\
\hline Pathological differentiation & & & & 0.775 & & & - \\
\hline Well differentiated & 83 & 1.000 & - & & - & - & \\
\hline $\begin{array}{l}\text { Moderately/poorly } \\
\text { differentiated }\end{array}$ & 18 & 1.083 & $0.627-1.869$ & & - & - & \\
\hline UICC pT factor & & & & 0.148 & & & - \\
\hline $\mathrm{T} 1 / \mathrm{T} 2$ & 7 & 1.000 & - & & - & - & \\
\hline $\mathrm{T} 3$ & 94 & 1.778 & $0.814-3.883$ & & - & - & \\
\hline Lymph node metastasis & & & & 0.074 & & & - \\
\hline N0 & 23 & 1.000 & - & & - & - & \\
\hline $\mathrm{N} 1$ & 78 & 1.597 & $0.956-2.669$ & & - & - & \\
\hline Lymphatic invasion & & & & 0.001 & & & 0.001 \\
\hline No & 46 & 1.000 & - & & 1.000 & - & \\
\hline Yes & 55 & 2.238 & $1.438-3.482$ & & 2.199 & $1.395-3.467$ & \\
\hline Vascular invasion & & & & 0.204 & & & - \\
\hline No & 38 & 1.000 & - & & - & - & \\
\hline Yes & 63 & 1.330 & $0.856-2.065$ & & - & - & \\
\hline hENT-1 status & & & & 0.046 & & & 0.049 \\
\hline High & 60 & 1.000 & - & & 1.000 & - & \\
\hline Low & 41 & 1.563 & $1.003-2.462$ & & 1.574 & $1.002-2.472$ & \\
\hline
\end{tabular}

hENT-1, human equilibrative nucleoside transporter 1; OR, odds ratio; CI, confidence interval; UICC, International Union Against Cancer.

A prognostic role of hENT-1 in pancreatic cancer has been also reported in previous studies. One of the largest retrospective studies demonstrating the predictive and prognostic value of hENT-1 used 229 specimens from the RTOG 9704 trial (15). The study randomized patients to gemcitabine or 5-FU treatment arms following pancreatic surgical resection; patients in both arms received concurrent chemotherapy with 5-FU and radiation. The results indicated that hENT-1 expression predicted OS and DFS in patients with pancreatic cancer who received gemcitabine, but not in those who received 5-FU, and the authors concluded that hENT-1 is a useful predictive biomarker of response to gemcitabine treatment, but not a prognostic biomarker (15). By contrast, Kim et al (22) investigated the prognostic value of hENT-1 and ribonucleoside reductase subunit M1 expression in 48 resected pancreatic cancer patients. The authors found that low expression of hENT-1 was associated with decreased OS and progression-free survival times in patients with resected pancreatic adenocarcinoma, independently of gemcitabine therapy. Therefore, future studies should focus on whether hENT-1 may have prognostic value as well as tpredictive value for sensitivity to gemcitabine.

Numerous studies have examined the presence and impact of hENT-1 protein overexpression or gene amplification in patients with pancreatic adenocarcinoma $(14,15)$. These studies have reported that hENT-1 is highly expressed in $40-80 \%$ of patients. The explanation for the variation in hENT-1 expression in the previous studies may be due to a number of reasons. First, different methods were used to examine the expression 
of hENT-1 in the previous studies: Spratlin et al (14) and Farrell et al (15) used immunohistochemistry performed on formalin-fixed, paraffin-embedded pancreatic tissue microarrays, whereas Giovannetti et al (13) used reverse transcription-polymerase chain reaction (RT-PCR) performed on laser-captured malignant cells from frozen biopsy or resection specimens, with results normalized to a single housekeeping gene (GAPDH), and the authors used RT-PCR performed on paraffin-embedded tissue with results normalized to two housekeeping genes (hydroxymethylbilane synthase and ribosomal protein L13a). Second, studies have investigated different stages of pancreatic cancer: Resectable pancreatic cancer was investigated in the present cohort and the Farrell et al study (15), whereas the study by Giovannetti et al (13) used a mixture of resectable cases and unresectable or recurrent cases of pancreatic cancer, and unresectable or recurrent pancreatic cancer cases were used in the study by Spratlin et al (14).

Regarding the associations between hENT-1 expression and clinicopathological factors in pancreatic cancer, the analysis by Farrell et al (15) of 538 patients who were assigned randomly to gemcitabine or 5-FU treatment groups following surgical resection indicated that tumor location in the pancreatic head (vs. all other locations) was the only baseline characteristic to have a positive statistical association between absent or positive hENT-1 expression in the gemcitabine treatment arm $(\mathrm{P}=0.02)$. By contrast, no positive statistical associations existed for this grouping between absent or positive hENT-1 expression in the 5-FU treatment arm. In the current study, a significant difference was observed only for sex, and there were no differences in any of the other clinicopathological parameters, including UICC pT factor and lymph node status, between the high and low hENT-1 expression groups; expression of hENT-1 appeared to be independent of other clinicopathological factors.

Caution is required when interpreting the current results, since the present study has several potential limitations. First, the present study was a retrospective analysis that was performed in a single institution. Therefore, there is a possibility that the present findings were observed by chance. Second, there was a selection bias in the patients in this series: Surgeons often avoid performing pancreatectomy in certain patients as the procedure is associated with high rates of morbidity and mortality (40-60\% and $1-1.5 \%$, respectively) (23-26); thus, the fact that certain patients in the present study received pancreatectomy may be considered to be a potential bias. In addition, Kanagawa Cancer Center is a specialized cancer center treating only cancer patients, and therefore, there is a possibility that only patients with good status were selected. Third, the evaluation of hENT-1 expression was not standardized, and the appropriate hENT-1 cutoff value is unclear. Considering these limitations, the results must be confirmed in another cohort or in a prospective multicenter study.

In summary, the OS and RFS rates of patients with pancreatic cancer who underwent curative resection followed by adjuvant gemcitabine chemotherapy differed significantly based on their hENT-1 expression. The present results indicate that gemcitabine may not be sufficient as a treatment, particularly for patients with low hENT-1 expression. These patients should be a target group for clinical trials of novel treatments.

\section{Acknowledgements}

The present study was supported, in part, by the Uehara Memorial Foundation (grant no. 201510145) and the Takeda Science Foundation.

\section{References}

1. Jemal A, Siegel R, Xu J and Ward E: Cancer statistics, 2010. CA Cancer J Clin 60: 277-300, 2010.

2. Nakao A, Fujii T, Sugimoto H, Kanazumi N, Nomoto S, Kodera Y, Inoue $\mathrm{S}$ and Takeda S: Oncological problems in pancreatic cancer surgery. World J Gastroenterol 12: 4466-4472, 2006.

3. Matsuno S, Egawa S, Fukuyama S, Motoi F, Sunamura M, Isaji S, Imaizumi T, Okada S, Kato H, Suda K, et al: Pancreatic cancer registry in Japan: 20 years of experience. Pancreas 28: 219-230, 2004.

4. Carpelan-Holmström M, Nordling S, Pukkala E, Sankila R, Lüttges J, Klöppel G and Haglund C: Does anyone survive pancreatic ductal adenocarcinoma? A nationwide study re-evaluating the data of the Finnish Cancer Registry. Gut 54: 385-387, 2005.

5. Wagner M, Redaelli C, Lietz M, Seiler CA, Friess H and Büchler MW: Curative resection is the single most important factor determining outcome in patients with pancreatic adenocarcinoma. Br J Surg 91: 586-594, 2004.

6. Neoptolemos JP, Stocken DD, Friess H, Bassi C, Dunn JA, Hickey H, Beger H, Fernandez-Cruz L, Dervenis C, Lacaine F, et al: A randomized trial of chemoradiotherapy and chemotherapy after resection of pancreatic cancer. N Engl J Med 350: 1200-1210, 2004.

7. Neoptolemos JP, Stocken DD, Bassi C, Ghaneh P, Cunningham D, Goldstein D, Padbury R, Moore MJ, Gallinger S, Mariette C, et al: Adjuvant chemotherapy with fluorouracil plus folinic acid vs gemcitabine following pancreatic cancer resection: A randomized controlled trial. JAMA 304: 1073-1081, 2010.

8. Oettle H, Post S, Neuhaus P, Gellert K, Langrehr J, Ridwelski K, Schramm H, Fahlke J, Zuelke C, Burkart C, et al: Adjuvant chemotherapy with gemcitabine vs observation in patients undergoing curative-intent resection of pancreatic cancer: A randomized controlled trial. JAMA 297: 267-277, 2007.

9. Zhang J, Visser F, King KM, Baldwin SA, Young JD and Cass CE: The role of nucleoside transporters in cancer chemotherapy with nucleoside drugs. Cancer Metastasis Rev 26: 85-110, 2007.

10. Mackey JR, Mani RS, Selner M, Mowles D, Young JD, Belt JA, Crawford CR and Cass CE: Functional nucleoside transporters are required for gemcitabine influx and manifestation of toxicity in cancer cell lines. Cancer Res 58: 4349-4357, 1998.

11. Pérez-Torras S, García-Manteiga J, Mercadé E, Casado FJ, Carbó N, Pastor-Anglada M and Mazo A: Adenoviral-mediated overexpression of human equilibrative nucleoside transporter 1 (hENT-1) enhances gemcitabine response in human pancreatic cancer. Biochem Pharmacol 76: 322-329, 2008.

12. Mori R, Ishikawa T, Ichikawa Y, Taniguchi K, Matsuyama R, Ueda M, Fujii Y, Endo I, Togo S, Danenberg PV and Shimada H: Human equilibrative nucleoside transporter 1 is associated with the chemosensitivity of gemcitabine in human pancreatic adenocarcinoma and biliary tract carcinoma cells. Oncol Rep 17: 1201-1205, 2007.

13. Giovannetti E, Del Tacca M, Mey V, Funel N, Nannizzi S, Ricci S, Orlandini C, Boggi U, Campani D, Del Chiaro M, et al: Transcription analysis of human equilibrative nucleoside transporter-1 predicts survival in pancreas cancer patients treated with gemcitabine. Cancer Res 66: 3928-3935, 2006.

14. Spratlin J, Sangha R, Glubrecht D, Dabbagh L, Young JD, Dumontet C, Cass C, Lai R and Mackey JR: The absence of human equilibrative nucleoside transporter 1 is associated with reduced survival in patients with gemcitabine-treated pancreas adenocarcinoma. Clin Cancer Res 10: 6956-6961, 2004.

15. Farrell JJ, Elsaleh H, Garcia M, Lai R, Ammar A, Regine WF, Abrams R, Benson AB, Macdonald J, Cass CE, et al: Human equilibrative nucleoside transporter 1 levels predict response to gemcitabine in patients with pancreatic cancer. Gastroenterology 136: 187-195, 2009.

16. Marechal R, Mackey JR, Lai R, Demetter P, Peeters M, Polus M, Cass CE, Young J, Salmon I, Devière J and Van Laethem JL: Human equilibrative nucleoside transporter 1 and human concentrative nucleoside transporter 3 predict survival after adjuvant gemcitabine therapy in resected pancreatic adenocarcinoma. Clin Cancer Res 15: 2913-2919, 2009. 
17. Sobin LH and Wittekind $\mathrm{CH}$ (eds): TNM Classification of Malignant Tumors. 7th edition. John Wiley \& Sons, New York, 2009.

18. Büchler MW, Friess H, Wagner M, Kulli C, Wagener V and Z'Graggen K: Pancreatic fistula after pancreatic head resection. Br J Surg 87: 883-889, 2000.

19. Wagner M, Z'graggen K, Vagianos CE, Redaelli CA, Holzinger F, Sadowski C, Kulli C, Zimmermann H, Baer HU and Büchler MW: Pylorus-preserving total pancreatectomy. Early and late results. Dig Surg 18: 188-195, 2001.

20. Andrén-Sandberg A, Wagner M, Tihanyi T, Löfgren P and Friess H: Technical aspects of left-sided pancreatic surgery for cancer. Dig Surg 16: 305-312, 1999.

21. Morinaga S, Nakamura Y, Watanabe T, Mikayama $H$, Tamagawa H, Yamamoto N, Shiozawa M, Akaike M, Ohkawa S, Kameda Y and Miyagi Y: Immunohistochemical analysis of human equilibrative nucleoside transporter-1 (hENT-1) predicts survival in resected pancreatic cancer patients treated with adjuvant gemcitabine monotherapy. Ann Surg Oncol 19 (Suppl 3): S558-S564, 2012.

22. Kim R, Tan A, Lai KK, Jiang J, Wang Y, Rybicki LA and Liu X: Prognostic roles of human equilibrative transporter 1 (hENT-1) and ribonucleoside reductase subunit M1 (RRM1) in resected pancreatic cancer. Cancer 117: 3126-3134, 2011.
23. Povoski SP, Karpeh MS Jr, Conlon KC, Blumgart LH and Brennan MF: Association of preoperative biliary drainage with postoperative outcome following pancreaticoduodenectomy. Ann Surg 230: 131-142, 1999.

24. Yeo CJ, Cameron JL, Lillemoe KD, Sohn TA, Campbell KA, Sauter PK, Coleman J, Abrams RA and Hruban RH: Pancreaticoduodenectomy with or without distal gastrectomy and extended retroperitoneal lymphadenectomy for periampullary adenocarcinoma, part 2: Randomized controlled trial evaluating survival, morbidity, and mortality. Ann Surg 236: 355-368, 2002.

25. Kawai M, Tani M, Terasawa H, Ina S, Hirono S, Nishioka R, Miyazawa M, Uchiyama K and Yamaue H: Early removal of prophylactic drains reduces the risk of intra-abdominal infections in patients with pancreatic head resection: Prospective study for 104 consecutive patients. Ann Surg 244: 1-7, 2006.

26. Munoz-Bongrand N, Sauvanet A, Denys A, Sibert A, Vilgrain V and Belghiti J: Conservative management of pancreatic fistula after pancreaticoduodenectomy with pancreaticogastrostomy. J Am Coll Surg 199: 198-203, 2004. 